\title{
A diffusive information preservation method for small Knudsen number flows
}

\author{
Fei Fei, Jing Fan* \\ State Key Laboratory of High Temperature Gas Dynamics, Institute of Mechanics, Chinese Academy of Sciences, Beijing 100190, China
}

\section{A R T I C L E I N F O}

\section{Article history:}

Received 24 July 2012

Received in revised form 21 February 2013

Accepted 3 March 2013

Available online 21 March 2013

\section{Keywords:}

DSMC

IP

Molecular diffusion

Time step

Cell size

\begin{abstract}
A B S T R A C T
The direct simulation Monte Carlo (DSMC) method is a powerful particle-based method for modeling gas flows. It works well for relatively large Knudsen (Kn) numbers, typically larger than 0.01 , but quickly becomes computationally intensive as Kn decreases due to its time step and cell size limitations. An alternative approach was proposed to relax or remove these limitations, based on replacing pairwise collisions with a stochastic model corresponding to the Fokker-Planck equation [J. Comput. Phys., 229, 1077 (2010); J. Fluid Mech., 680, 574 (2011)]. Similar to the DSMC method, the downside of that approach suffers from computationally statistical noise. To solve the problem, a diffusion-based information preservation (D-IP) method has been developed. The main idea is to track the motion of a simulated molecule from the diffusive standpoint, and obtain the flow velocity and temperature through sampling and averaging the IP quantities. To validate the idea and the corresponding model, several benchmark problems with $\mathrm{Kn} \sim 10^{-3}-10^{-4}$ have been investigated. It is shown that the IP calculations are not only accurate, but also efficient because they make possible using a time step and cell size over an order of magnitude larger than the mean collision time and mean free path, respectively.
\end{abstract}

(c) 2013 Elsevier Inc. All rights reserved.

\section{Introduction}

Numerical schemes can be categorized into two kinds: continuum and particle. For real gas flows, a powerful molecular approach is the direct simulation Monte Carlo (DSMC) method [1] that has been successfully applied to various rarefied gas flows [2], especially hypersonic situations, e.g. the structures of strong shock waves [3], aerodynamic features of reentry vehicles [4]. There is a great interest to extend the DSMC method to other situations not only for understanding the mechanisms and patterns of gas flows at molecular level, but also for sometimes more conveniently and physically modeling the microscopic transport process behind macroscopic flow phenomena.

To achieve the goal, an issue that has to be addressed firstly is the limitations of the cell size and time step for the DSMC method $[5,6]$. They become stringent when the temporal and spatial scales of a gas flow are much larger than those of molecular motion.

The time step limitation of DSMC results from a core assumption that decouples molecular motion and collisions in a time step $\Delta t$ [1]. It is physically reasonable only when $\Delta t<\tau_{c}$, where $\tau_{c}$ is the mean collision time of molecules. Another essential feature of DSMC [1] is to select collision pairs based on pairs of molecules, which is reasonable only when the distance between a pair of molecules is smaller than the mean free path $(\lambda)$. In the conventional DSMC method, pairs of molecules are formed within a cell, and therefore the cell size needs to be smaller than $\lambda$. The values of $\tau_{c}$ and $\lambda$ are usually very small,

\footnotetext{
* Corresponding author. Tel.: +8610 82543880 .

E-mail address: jfan@imech.ac.cn (J. Fan).
} 
e.g. about $2 \times 10^{-10} \mathrm{sec}$ and $6 \times 10^{-8} \mathrm{~m}$ for air in the standard conditions, respectively. Consequently, it is hard to meet the requirements even for parallel supercomputers, except for relatively large Knudsen number flows where the characteristic scales are comparable to or less than $\tau_{c}$ and $\lambda$.

Many efforts were made to release the time step and cell size limitations for DSMC. One of them tracked the motion of a simulated molecule (simulator) in a diffusion viewpoint instead of the decoupling treatment of molecular motion and collisions $[7,8]$. It to certain extent eliminated the sources that results in the limitations of time step and collision-pairs distance in DSMC. Along this way, a critical point is how to evaluate flow velocity and temperature that are necessary to calculate the convective motion and diffusive motion of simulators every time step. In previous studies [7,8], they were obtained through sampling and averaging microscopic velocities. As pointed out by the authors themselves, "stochastic noise will be the same for the new model and DSMC", and "difficulties due to noise for low Mach number flows can be expected for both methods".

In this paper, we suggest to combine the diffusion approach with the information preservation (IP) method. The IP method $[9,10]$ was originally proposed to overcome the statistical scatter in DSMC calculating microscale gas flows in MEMS usually in low-speed. IP assigns each simulated molecule two velocities: one is microscopic velocity, $c_{k, i}$ like DSMC, and the other is called information velocity, $u_{k, i}$, that can be understood as the collective velocity of a large number of real molecules represented by a simulator. In conventional IP method, molecular motion is tracked based on $c_{k, i}$ following the same steps as DSMC, and $u_{k, i}$ is transported along with molecular motion. When a simulator encounters a computational boundary during its movement, $u_{k, i}$ takes the boundary value. When two simulators collide, their information velocities are exchanged according to the corresponding models. The flow velocity is obtained through sampling and averaging the information velocities, which avoids the statistical scatter arising from the random part of the microscopic velocities. The IP method has been successfully applied many situations, e.g. unidirectional flows [9,10], microchannel flows [11,12], and micro plate aerodynamics $[13,14]$. All of them exhibited a good agreement with exact solutions or measured data available. A recent advancement of IP was a clear theoretical verification that was based on Maxwell's equation of transfer and established the governing equation of information quantities [15-17].

Briefly speaking, our idea is to track the motion of simulated molecules in a diffusive view, and obtain the flow velocity and temperature through the IP quantities. In this paper, a diffusion model in consistence with the IP method is described. Prior to it, the Langevin equation is briefly reviewed that is a basis to set up the diffusion model. Then several benchmark problems with small Knudsen numbers are calculated using the present IP method, and the IP results are verified in comparison with the DSMC and Navier-Stokes solutions.

\section{The Langevin equation}

It is well known that the Langevin equation was originally invented to explain the Brownian motion of dust particles, which can be written as [18-20]

$$
\begin{aligned}
& \frac{d r_{i}}{d t}=c_{i}, \\
& \frac{d c_{i}}{d t}=-\zeta c_{i}+A_{i},
\end{aligned}
$$

where $r_{i}$ and $c_{i}$ are the particle position and velocity, $\zeta c_{i}$ and $A_{i}$ are the mean and fluctuating forces acted on the particle by the surrounding molecules, $\zeta=k_{B} T /(m D), k_{B}$ is the Boltzmann constant, and $m, D$ and $T$ are the molecular mass, diffusion coefficient, and temperature of the ambient fluid, respectively.

Eq. (1) is a stochastic differential equation, and its solution describes the position and velocity evolution of a particle in a probabilistic sense. For locally isotropic cases, Eq. (1) has an analytical solution that was firstly obtained by Chandrasekhar [18]

$$
W\left(R_{x}, S_{x}\right)=\frac{1}{2 \pi\left(F G-H^{2}\right)^{1 / 2}} \exp \left[-\frac{R_{x}^{2}}{2 F}-\frac{\left(F S_{x}-H R_{x}\right)^{2}}{2 F\left(F G-H^{2}\right)}\right],
$$

with

$$
\begin{aligned}
& R_{x}=x-x_{0}-c_{x 0}\left(1-e^{-\zeta t}\right) / \zeta, \\
& S_{x}=c_{x}-c_{x 0} e^{-\zeta t}, \\
& F=\frac{k_{B} T}{m \zeta^{2}}\left(4 e^{-\zeta t}-e^{-2 \zeta t}+2 \zeta t-3\right), \\
& G=\frac{k_{B} T}{m}\left(1-e^{-2 \zeta t}\right),
\end{aligned}
$$




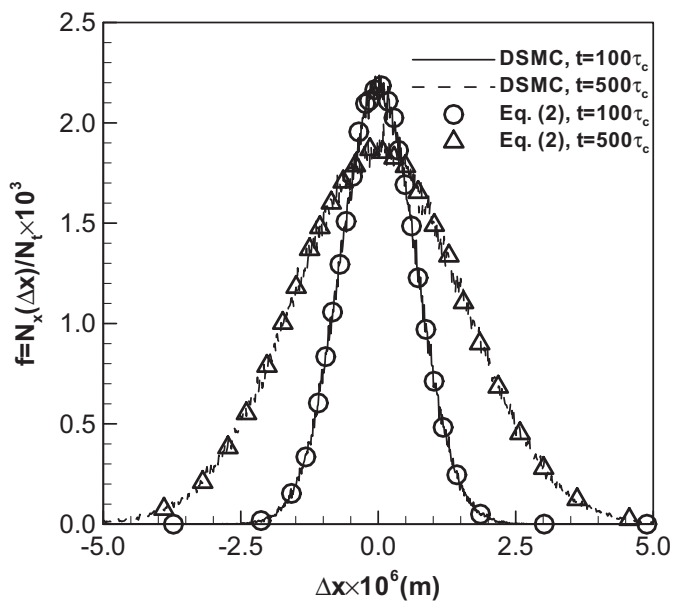

Fig. 1. Comparison of the molecular position distributions at two time intervals. Symbols: the Chandrasekhar distribution (2); lines: the DSMC results.

$$
H=\frac{k_{B} T}{m \zeta}\left(1-e^{-\zeta t}\right)^{2},
$$

where $x_{0}$ and $c_{x 0}$ are the initial position coordinate and velocity component in the $x$ direction, respectively.

As discussed in details in [18-20], the Langevin equation (1) not only applies to dust particles, but also many other phenomena. For molecular motion interested here, when time is much larger than $\tau_{c}$, the force acted on a molecule can also be divided into two parts: mean and fluctuating. As a result, the Chandrasekhar distribution (2) is also suitable to describe the position and velocity evolution of a molecule. A numerical comparison is made in Fig. 1 for an argon gas in the standard condition. Initially a group of molecules gathered at $x=0$, with a temperature of $273 \mathrm{~K}$ and zero flow velocity. We see clearly that the molecular position distributions at two time intervals ( $100 \tau_{c}$ and $500 \tau_{c}$ ) predicted by (2) agree well with the DSMC results.

\section{A diffusive information preservation method}

In a diffusive viewpoint, molecular motion can be expressed as follows

$$
r_{k, i}^{t+\Delta t}=r_{k, i}^{t}+u_{k, i}^{t} \times \Delta t+\Delta r_{k, i}^{D}
$$

where the second and third terms on the right-side hand reflect the convective motion and diffusive motion of a molecule, respectively.

If we employ the Langevin equation to describe the diffusive motion of a molecule, according to the Chandrasekhar distribution (2), we have

$$
\begin{aligned}
& \Delta r_{k, i}^{D}=\left(-2 F \ln \Phi_{r}\right)^{1 / 2} \cos \alpha_{r}+c_{k, i}^{\prime}(t)[1-\exp (-\zeta \Delta t)] / \zeta, \\
& c_{k, i}^{\prime}(t+\Delta t)=\left[-\frac{2\left(F G-H^{2}\right)}{F} \ln \Phi_{r}\right]^{1 / 2} \cos \alpha_{r}+\frac{H \times R_{i}}{F}+c_{k, i}^{\prime}(t) \exp (-\zeta \Delta t),
\end{aligned}
$$

where $\Phi_{r}$ and $\alpha_{r}$ are random numbers uniformly distributed in $[0,1]$ and $[0,2 \pi]$, respectively.

A diffusive information preservation (D-IP) method suggests to track the motion of a simulated molecule using Eqs. (4)(6), calculate the convective motion in Eq. (4) based on the information velocity, and obtain temperature used in Eqs. (5) and (6) through sampling and averaging the information temperature every time step. Since the Chandrasekhar distribution is based on a local equilibrium assumption, the time step of D-IP calculations has to be small enough in comparison with the characteristic flow time.

Similar to the conventional IP method, there are six steps to implement the D-IP method.

1. Initialization. Each simulator is initially given a position $r_{k, i}(k=1,2$, ldots, $N)$, a thermal velocity $c_{k, i}^{\prime}$, an information velocity $u_{k, i}$, and an information temperature $T_{I P-k}$ according to the initial conditions of a gas flow.

2. Molecular motion. After a time step, simulator $k$ acquires a new position and thermal velocity according to Eqs. (4)-(6).

3. Update information velocity and temperature. After a time step, the information velocity and temperature of simulator $k$ is updated according to their governing equations derived as follows. 


\subsection{Maxwell's equation of change}

The governing equations of information velocity and temperature have been derived based on Maxwell's transport equation in Ref. [15-17]. We will follow a similar means to investigate the differences arising from the diffusion model to replace the molecular motion model in the conventional IP method.

The conservation form of Maxwell's equation of change can be written as

$$
\frac{\partial}{\partial t}(n \bar{Q})+\frac{\partial}{\partial x_{j}}\left(n \overline{c_{j} Q}\right)=\Delta[Q]
$$

where the collision term $\Delta[Q]$ is a weighted average of the change in the total value of molecular properties $Q$ before and after collisions, and the external force term is neglected.

For clarity, the same notations as [15] are explained: the microscopic velocity $c_{i}$, the information velocity $u_{i}$, the macroscopic velocity of the flow field $U_{i}$, and the information temperature $T_{I P}$. They are connected through velocity fluctuations: $c_{i}^{\prime}=c_{i}-u_{i}, c_{i}^{\prime \prime}=u_{i}-U_{i}, c_{i}^{\prime \prime \prime}=c_{i}-U_{i}$, and $\overline{c^{2}}=\overline{3 R T_{I P}+u^{2}}$.

If $Q$ is set as momentum $m c_{i}$ and translational energy $m c^{2} / 2$, then Eq. (7) reads as,

$$
\begin{aligned}
& \frac{\partial}{\partial t}\left(n m \overline{c_{i}}\right)+\frac{\partial}{\partial x_{j}}\left(n m \overline{c_{i} c_{j}}\right)=\Delta\left[m c_{i}\right] \\
& \frac{\partial}{\partial t}\left(\frac{1}{2} n m \overline{c^{2}}\right)+\frac{\partial}{\partial x_{j}}\left(\frac{1}{2} n m \overline{c_{j} c^{2}}\right)=\Delta\left[\frac{1}{2} m \overline{c^{2}}\right] .
\end{aligned}
$$

It can easily shown that $\overline{c_{i} c_{j}}=\overline{u_{i} u_{j}}+\overline{u_{i} c_{j}^{\prime}}+\overline{c_{i}^{\prime} c_{j}^{\prime \prime \prime}}$, and $\overline{c_{j} c^{2}}=\overline{c_{j}\left(3 R T_{I P}+u^{2}\right)}-\overline{c_{j}^{\prime \prime \prime}\left(3 R T_{I P}+u^{2}-c^{2}\right)}$. Substituting them into Eqs. (8) and (9), respectively, we have

$$
\begin{aligned}
& \frac{\partial}{\partial t}\left(n m \overline{u_{i}}\right)+\frac{\partial}{\partial x_{j}}\left(n m \overline{u_{i} u_{j}}\right)=-\frac{\partial}{\partial x_{j}}\left(n m \overline{u_{i} c_{j}^{\prime}}\right)-\frac{\partial}{\partial x_{j}}\left(n m \overline{c_{i}^{\prime} c_{j}^{\prime \prime \prime}}\right)+\Delta\left[m c_{i}\right], \\
& \frac{\partial}{\partial t}\left(n m \overline{\left(3 R T_{I P}+u^{2}\right)}\right)+\frac{\partial}{\partial x_{j}}\left(n m \overline{u_{j}\left(3 R T_{I P}+u^{2}\right)}\right)=-\frac{\partial}{\partial x_{j}}\left(n m \overline{c_{j}^{\prime}\left(3 R T_{I P}+u^{2}\right)}\right)+\frac{\partial}{\partial x_{j}}\left(n m \overline{c_{j}^{\prime \prime \prime}\left(3 R T_{I P}+u^{2}-c^{2}\right)}\right)+\Delta\left[m \overline{c^{2}}\right]
\end{aligned}
$$

Equation (10) governs the evolution of the average of the information velocity. The first term on the left hand side is the change rate of the momentum. The second term is the changes due to the collective movement corresponding to $u_{k, i}^{t} \times \Delta t$ in the diffusion model (4). On the right hand side, the first term is the change due to the thermal movement corresponding to $\Delta r_{k, i}^{D}$ in the diffusion model (4). The second term is a microscopic correlation term, and the final term is the collision integral term. The meaning of each term in Eq. (11) is similar to the corresponding one in Eq. (10).

\subsection{Individual information velocity and temperature}

To apply Eqs. (10) and (11) to individual simulator, two assumptions suggested in [15] are employed here. One assumed that a similar connection applies to an individual molecule in the IP method, and the other assumes that the correlation terms apply equally to individual molecules. Then, the governing equation for individual information velocity and temperature are expressed as follows:

$$
\begin{aligned}
\frac{\partial}{\partial t}\left(n m u_{i}\right)+\text { collective movement }=-\frac{\partial}{\partial x_{j}}\left(n m \overline{u_{i} c_{j}^{\prime}}\right)-\frac{\partial}{\partial x_{j}}\left(n m \overline{c_{i}^{\prime} c_{j}^{\prime \prime \prime}}\right)+\text { collisions } \\
\begin{aligned}
\frac{\partial}{\partial t}\left[n m\left(3 R T_{I P}+u^{2}\right)\right]+\text { collective movement }= & -\frac{\partial}{\partial x_{j}}\left(n m \overline{3 R T_{I P} c_{j}^{\prime}}\right)-\frac{\partial}{\partial x_{j}}\left(n m \overline{u^{2} c_{j}^{\prime}}\right)+\frac{\partial}{\partial x_{j}}\left(n m \overline{c_{j}^{\prime \prime \prime}\left(3 R T_{I P}+u^{2}-c^{2}\right)}\right) \\
& + \text { collisions. }
\end{aligned}
\end{aligned}
$$

\subsection{Correlated terms $\overline{c_{i}^{\prime} c_{j}^{\prime \prime \prime}}$ and $\overline{c_{j}^{\prime \prime \prime}\left(3 R T_{I P}+u^{2}-c^{2}\right)}$}

As discussed in details in [15-17], there are two means to evaluate the correlation terms, namely a local equilibrium approach and a flux splitting approach. In situations with small Knudsen numbers, because of the temporal and spatial scales much larger than those of molecular motion, the local equilibrium condition prevails, and the correlated terms $\overline{c_{i}^{\prime} c_{j}^{\prime \prime \prime}}$ and $\overline{c_{j}^{\prime \prime \prime}\left(3 R T_{I P}+u^{2}-c^{2}\right)}$ can be simplified as follows [15-17]

$$
n m \overline{c_{i}^{\prime} c_{j}^{\prime \prime \prime}}=p,
$$

$$
n m \overline{c_{j}^{\prime \prime \prime}\left(3 R T_{I P}+u^{2}-c^{2}\right)}=-2 p U_{j} .
$$




\subsection{Correlated terms $\overline{u_{i} c_{j}^{\prime}}$ and $\overline{c_{j}^{\prime}\left(3 R T_{I P}+u^{2}\right)}$}

Because the information velocity remains unchanged during the diffusive motion of a simulator, the momentum at certain spatial point $M_{i}\left(r_{j}, t\right)=m u_{i}\left(r_{j}, t\right) n\left(r_{j}, t\right)$ will change only due to the number density, $n\left(r_{j}, t\right)$, while $n\left(r_{j}, t\right)$ evolves by [18],

$$
n\left(r_{j}, t+\Delta t\right)=\int_{-\infty}^{+\infty} n\left(r_{j}-\Delta r_{j}, t\right) w\left(\Delta r_{j}, \Delta t\right) d\left(\Delta r_{j}\right)
$$

where $w\left(\Delta r_{j}, \Delta t\right)$ is the probability distribution of molecular position after moving through a time interval $\Delta t$. [18],

Under a local equilibrium condition, $w\left(\Delta r_{j}, \Delta t\right)$ for a diffusive process governed by Langevin equation (1) can be written as

$$
w\left(\Delta r_{j}, \Delta t\right)=\frac{1}{\left(4 \pi D^{*} \Delta t\right)^{3 / 2}} \exp \left(-\frac{\Delta r^{2}}{4 D^{*} \Delta t}\right)
$$

with $D^{*}=\frac{k_{B} T}{m \zeta^{2}}\left[\zeta-\left(1-e^{-\zeta \Delta t}\right) / \Delta t\right]$.

The momentum change due to molecular diffusion (16) is

$$
M_{i}\left(r_{j}, t+\Delta t\right)=\int_{-\infty}^{+\infty} M_{i}\left(r_{j}-\Delta r_{j}, t\right) w\left(\Delta r_{j}, \Delta t\right) d\left(\Delta r_{j}\right) .
$$

Substituting Eq. (17) into (18) and making Taylor's expansion for $M_{i}\left(r_{j}-\Delta r_{j}, t\right)$, we have

$$
M_{i}\left(r_{j}, t+\Delta t\right)=M_{i}\left(r_{j}, t\right)+D^{*} \Delta t\left(\frac{\partial^{2} M_{i}}{\partial x^{2}}+\frac{\partial^{2} M_{i}}{\partial y^{2}}+\frac{\partial^{2} M_{i}}{\partial z^{2}}\right)+O\left(\Delta t^{2}\right) .
$$

As $\Delta t \rightarrow 0$, Eq. (19) gives rise to

$$
\frac{\partial\left(\rho u_{i}\right)}{\partial t}=\lim _{\Delta t \rightarrow 0} \frac{M_{i}\left(r_{j}, t+\Delta t\right)-M_{i}\left(r_{j}, t\right)}{\Delta t}=\rho D^{*} \frac{\partial^{2} u_{i}}{\partial x_{j} \partial x_{j}},
$$

since $\rho D^{*} \approx \rho D=6 \mu / 5$ under a hard-sphere model for molecular interaction.

According to classical fluid mechanics, the momentum change due to viscosity is

$$
\frac{\partial\left(\rho u_{i}\right)}{\partial t}=\mu \frac{\partial^{2} u_{i}}{\partial x_{j} \partial x_{j}}+\frac{\mu}{3} \frac{\partial}{\partial x_{i}}\left(\frac{\partial u_{j}}{\partial x_{j}}\right) .
$$

In the continuum limit, the right hand sides of Eqs. (20) and (21) should be equal. To satisfy the requirement, the first term on the right hand side of Eq. (12) needs to be modified as follows,

$$
I_{u}=-\psi_{\eta} \frac{\partial}{\partial x_{j}}\left(n m \overline{u_{i} c_{j}^{\prime}}\right)-\frac{\partial}{\partial x_{i}}\left(\frac{1}{3} \psi_{\eta} n m \overline{u_{j} c_{j}^{\prime}}\right),
$$

with $\psi_{\eta}=\mu /\left(\rho D^{*}\right)$.

For temperature, similar to the derivations (16)-(22), we have

$$
\frac{\partial(\rho T)}{\partial t}=\rho D^{*} \frac{\partial^{2} T}{\partial x_{j} \partial x_{j}} .
$$

According to fluid mechanics, the temperature change due to thermal conductivity is

$$
\frac{\partial(\rho T)}{\partial t}=\frac{\kappa}{c_{v}} \frac{\partial^{2} T}{\partial x_{j} \partial x_{j}} .
$$

In the continuum limit, the right hand sides of Eqs. (23) and (24) should be equal also. To satisfy the requirement, the first term on the right hand side of Eq. (13) needs a following modification

$$
I_{T-\kappa}=-\psi_{\kappa} \frac{\partial}{\partial x_{j}}\left(n m \overline{3 R T_{I P} C_{j}^{\prime}}\right)
$$

with $\psi_{\kappa}=\kappa /\left(\rho c_{v} D^{*}\right)$.

The second term on the right hand side of Eq. (13) reflects the energy change due to viscosity. Following a similar analysis to Eqs. (16)-(22), we have

$$
I_{T-\mu}=-\psi_{\eta} \frac{\partial}{\partial x_{j}}\left(n m \overline{u^{2} c_{j}^{\prime}}\right)-\frac{2}{3} \psi_{\eta} \frac{\partial}{\partial x_{j}}\left(n m \bar{u}_{j} \overline{c_{i}^{\prime} u_{i}}\right),
$$

Substituting Eqs. (14) and (22) into (12), and Eqs. (15), (25) and (26) into (13), yields the governing equations of information velocity and temperature as follows, which are consistent with the diffusion model (4)-(6). 


$$
\begin{aligned}
& \frac{\partial}{\partial t}\left(n m u_{i}\right)+\text { collective movement }=-\psi_{\eta} \frac{\partial}{\partial x_{j}}\left(n m \overline{u_{i} c_{j}^{\prime}}\right)-\frac{\partial}{\partial x_{i}}\left(\frac{1}{3} \psi_{\eta} n m \overline{u_{j} c_{j}^{\prime}}\right)-\frac{\partial p}{\partial x_{i}}+\text { collisions }, \\
& \frac{\partial}{\partial t}\left[n m\left(3 R T_{I P}+u^{2}\right)\right]+\text { collective movement }=-\psi_{\kappa} \frac{\partial}{\partial x_{j}}\left(3 R n m \overline{T_{I P} c_{j}^{\prime}}\right)-\psi_{\eta} \frac{\partial}{\partial x_{j}}\left(n m \overline{u^{2} c_{j}^{\prime}}\right)-\frac{2}{3} \psi_{\eta} \frac{\partial}{\partial x_{j}}\left(n m U_{j} \overline{c_{i}^{\prime} u_{i}}\right) \\
& -2 \frac{\partial}{\partial x_{j}}\left(p U_{j}\right)+\text { collisions }
\end{aligned}
$$

4. Information velocity and temperature changes due to collisions. A time step of D-IP is usually much larger than the mean collision time. This means that a simulator will experience enough collisions during it. Therefore, it is assumed that the information velocity and temperature at $t+\Delta t$ is equal to the mean values of its surrounding molecules, i.e.

$$
\begin{aligned}
u_{k, i}^{t+\Delta t} & =\frac{1}{N_{k}} \sum_{j=1}^{N_{k}} u_{j, i}^{t+\Delta t}, \\
T_{I P-k}^{t+\Delta t} & =\frac{1}{N_{k}} \sum_{j=1}^{N_{k}} T_{I P-j}^{t+\Delta t}+\frac{\psi_{\eta}}{2 c_{v} N_{k}}\left(\sum_{j=1}^{N_{k}} u_{j}^{t 2}-\sum_{j=1}^{N_{k}} u_{j}^{t+\Delta t 2}\right)
\end{aligned}
$$

where $N_{k}$ is the number of simulators in a neighboring region around simulator $k$, the region size can be chosen according to the spatial scales and computational accuracy of flows, and $c_{V}$ is the specific heat at constant volume. The second term on the right-hand side of Eq. (30) is necessary to satisfy the energy conservation and viscous dissipation.

5. Interact with boundaries. When a simulator encounters a computational boundary during its movement in a time step, its information velocity and temperature take the boundary values as usual. A criterion whether it interacts with a computational boundary is described in details in Appendix.

6. Sample and average. The flow velocity and temperature are obtained by sampling the information quantities of simulators each cell through ensemble average for unsteady cases, and time average for steady cases, i.e.

$$
\begin{aligned}
& U_{i}=\overline{u_{i}}, \\
& T_{c}=\overline{T_{I P}} .
\end{aligned}
$$

The flow number density is calculated using the mass conservation equation:

$$
\frac{\partial(n m)}{\partial t}+\frac{\partial\left(n m U_{j}\right)}{\partial x_{j}}=0
$$

\section{Benchmark problems}

\subsection{Couette flow}

The Couette flow is a steady flow driven by two infinite and parallel plates moving oppositely along their planes. In the present study, the flow medium is argon gas in the standard condition ( $1 \mathrm{~atm}$ and $273 \mathrm{~K}$ ), and the plates move oppositely at speed of $50 \mathrm{~m} / \mathrm{s}$, respectively, whose surfaces are diffusively reflecting and have the same temperature as the gas. The distance between the plates $H=500 \lambda$.

Three calculations are performed using the D-IP method. All of them start from a stationary uniform flow field. Different time steps and cell sizes are employed, which are $10 \tau_{c}$ and $10 \lambda$ for the first calculation, $20 \tau_{c}$ and $20 \lambda$ for the second, and $30 \tau_{c}$ and $25 \lambda$ for the third. As shown in Fig.2, the velocity profiles of D-IP all agree well with the Navier-Stokes exact solution $U /$ $U_{w}=2 y / H-1$. The surface shear stresses $\left(\mathrm{N} / \mathrm{m}^{2}\right)$ obtained by the three D-IP calculations are $67.0,66.8$ and 66.8, respectively, in excellent agreement with value of 67.6 predicted by the Navier-Stokes exact solution $\tau_{w}=2 \mu U_{w} / H$.

\subsection{Poiseuille flow}

The Poiseuille flow is a steady flow confined between two infinite and parallel plates. It is driven by a pressure gradient along the plates, $d p / d x$. In the present study, $d p / d x=\alpha p_{0} / H, a=-2.54 \times 10^{-3}, p_{0}=1 \mathrm{~atm}$, the distance between the plates $H=500 \lambda$, while the other computational parameters are the same as those used in the Couette flow.

As shown in Fig.3, the velocity profiles of D-IP from the center to the upper plate in the three calculations all agree well with the Navier-Stokes exact solution of the equation $U=-d p / d x \times\left(H y-y^{2}\right) /(2 \mu)$. The values of the surface shear stress acting on the upper plate $\left(\mathrm{N} / \mathrm{m}^{2}\right)$ obtained by the D-IP calculations are all 129 , and they compare well with the value of 128.7 given by the $\mathrm{N}-\mathrm{S}$ exact solution $\tau_{w}=0.5 \alpha p_{0}$. 


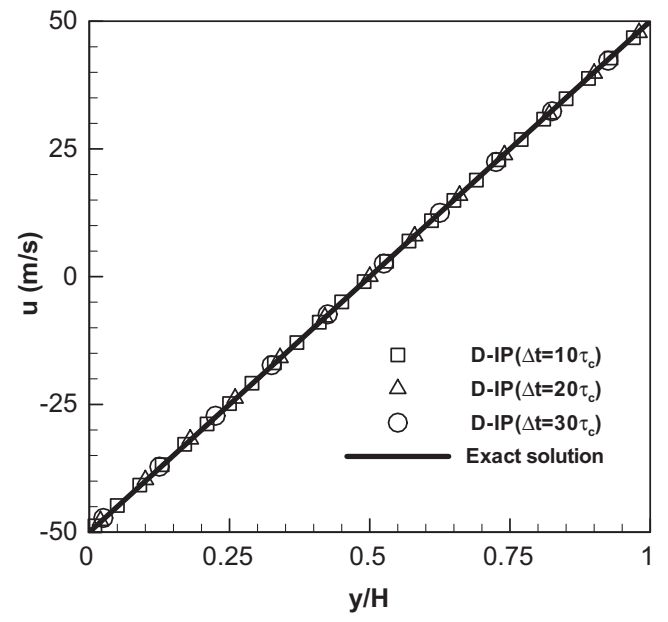

Fig. 2. Velocity profiles obtained by the D-IP method for Couette flow $(\mathrm{Kn}=0.002)$ versus the Navier-Stokes exact solution.

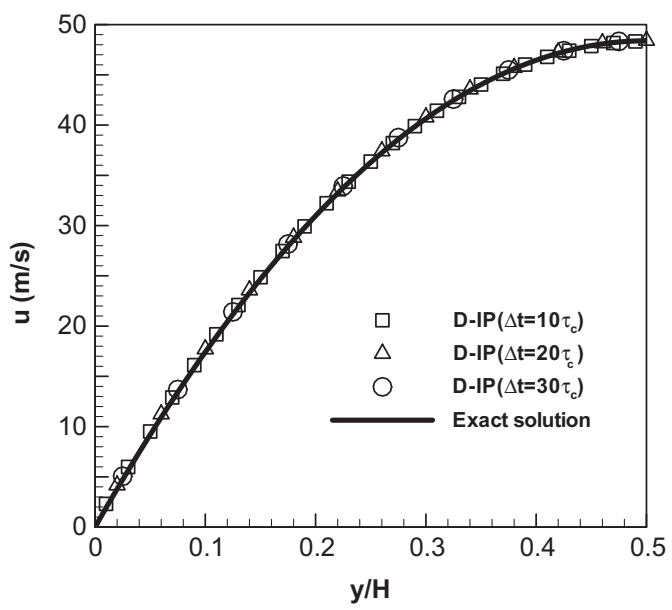

Fig. 3. Velocity profiles obtained by the D-IP method for Poiseuille flow $(\mathrm{Kn}=0.002)$ versus the Navier-Stokes exact solution.

\subsection{Rayleigh flow}

In the Rayleigh flow, the stationary plate acquires a speed of $U_{w}$ along the plate direction at the initial time $(t=0)$. The impulsive motion induces an unsteady flow of the gas near the plate, for which an ensemble average has to be used in the D-IP calculation. In the present study, $U_{w}=50 \mathrm{~m} / \mathrm{s}$, and the outer boundary is placed $500 \lambda$ away from the plate to avoid possible backward disturbance to the flow evolution near the plate during the time interval of the calculation. The time step and cell size of D-IP are $10 \tau_{c}$ and $10 \lambda$, respectively. The other computational parameters are the same as those used in the Couette flow.

Fig. 4 shows the velocity profile at $500 \tau_{c}$, as well as the surface shear stress acting on the plate versus time, obtained by the D-IP method. They agree well with the Navier-Stokes exact solutions

$$
U / U_{w}=1-\operatorname{erf}(y / \sqrt{4 v t})
$$

and

$$
\tau_{w}=\mu U_{w} / \sqrt{\pi v t}
$$

respectively, where erf denotes an error function, and $v=\mu / \rho$. 


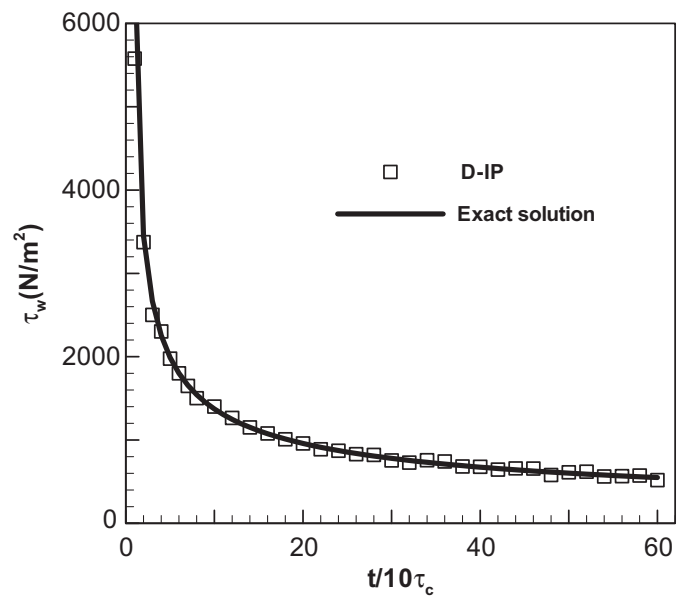

(a) Surface shear stress versus time

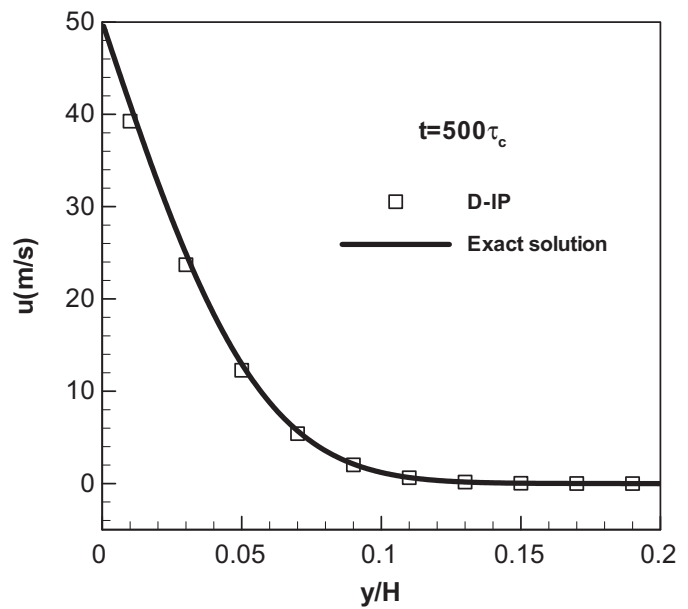

(b) Velocity profiles at $t=500 \tau_{c}$

Fig. 4. Evolution of the surface shear stress and flow velocity predicted by the D-IP method and the Navier-Stokes exact solutions for the Rayleigh flow.

\subsection{Thermal Couette flow}

Thermal Couette flow is driven by a temperature difference between two infinite and parallel plates. In the present study, the bottom and top plates have temperatures of $300 \mathrm{~K}$ and $273 \mathrm{~K}$, respectively, while the other computational parameters are the same as those used in the Couette flow in Section 4.1.

As shown in Fig. 5, the number density and temperature profiles of D-IP in the three calculations all agree well with that obtained by the DSMC method, so do the values of the heat flux $\left(\mathrm{W} / \mathrm{m}^{2}\right)$ given in the fourth row of Table 1 . Note that in Table 1 the CPU hours of Intel i5 $2.66 \mathrm{GHz}$ consumed by the D-IP calculations decrease as the time steps and cell sizes increase, and all of them are much less than that of DSMC.

\subsection{Thermal Rayleigh flow}

In a thermal Rayleigh flow, the stationary plate acquires a temperature increase $\Delta T_{w}$ at the initial time $(t=0)$. This induces an unsteady flow of the gas near the plate. In the present study, $\Delta T_{w}=27 \mathrm{~K}$, and the outer boundary is placed $500 \lambda$ away from the plate to avoid possible backward disturbance during the calculted time interval. The time step and cell size of D-IP are $10 \tau_{c}$ and $10 \lambda$, respectively. The other computational parameters are the same as those used in the Couette flow.

Fig. 6 shows the flow fields at two moments $\left(300 \tau_{c}\right.$ and $\left.500 \tau_{c}\right)$, as well as the heat flux versus time, obtained by the D-IP method and the DSMC method. They agree well with each other, except the heat flux at the beginning interval where the time step used in D-IP is too large to resolve the evolving details (Fig. 6d). Due to the time step and cell size used in the D-IP calculation are much larger than those in the DSMC one, together with the intrinsic advantage of the IP method that 


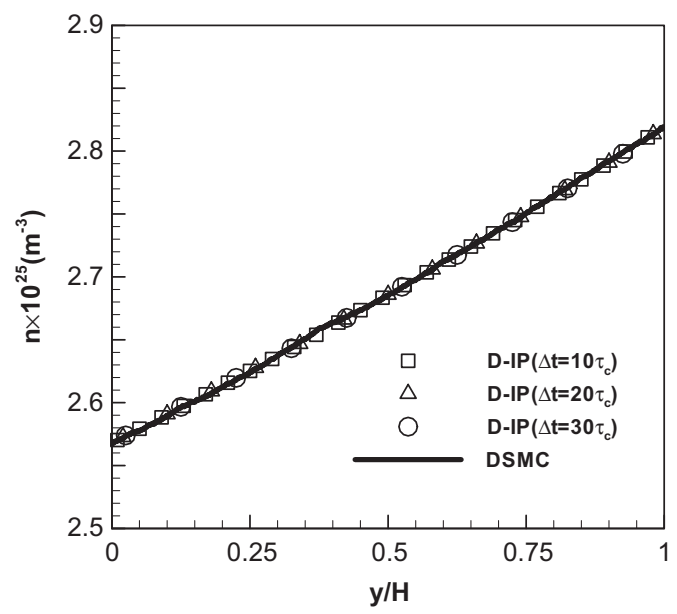

(a) Number density

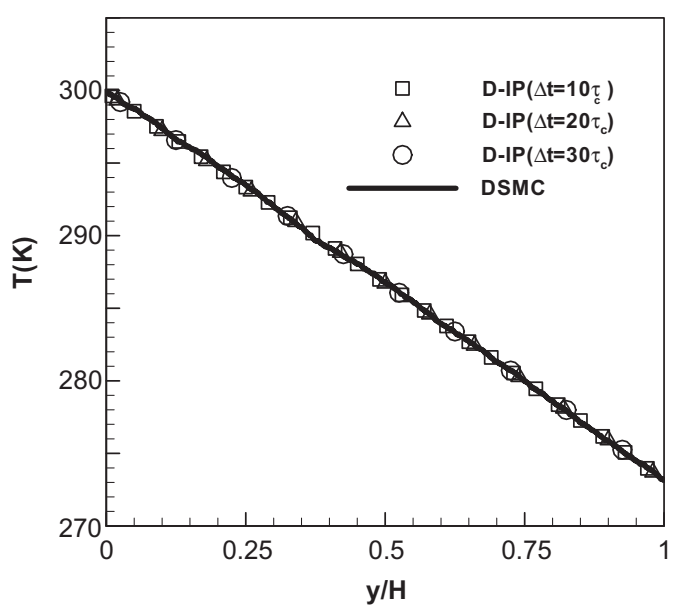

(b) Temperature

Fig. 5. Comparison of flow field profiles obtained by D-IP and DSMC for thermal Couette flow $(\mathrm{Kn}=0.002)$.

Table 1

Parameters and results of D-IP and DSMC calculating thermal Couette flow.

\begin{tabular}{lllll}
\hline Parameters & $\Delta t$ & $\Delta y$ & $q_{w}\left(\mathrm{~W} / \mathrm{m}^{2}\right)$ & CPU (hr) \\
\hline D-IP & $10 \tau_{c}$ & $10 \lambda$ & $1.46 \times 10^{4}$ & 0.27 \\
& $20 \tau_{c}$ & $20 \lambda$ & $1.45 \times 10^{4}$ & 0.1 \\
DSMC & $30 \tau_{c}$ & $25 \lambda$ & $1.45 \times 10^{4}$ & 0.08 \\
\hline
\end{tabular}

reaches a statistical convergence at a normal sampling size (usually $10^{3}-10^{4}$ ) even for quite low Mach number flows, the former consumed the CPU hour greatly less than the latter. In this test case, the D-IP calculation took about 0.05 CPU hour on a computer with an Intel i5 $2.66 \mathrm{GHz}$, while the DSMC calculation, carried out on a parallel computer, took about 7000 CPU hours if converted to the Intel i5, five order of magnitude more than the D-IP.

\subsection{Square cavity flow}

A square cavity flow is a steady flow driven by the lid side moving at speed of $U_{\text {lid }}$ along the plate direction (Fig. 7), while the other three sides keep at rest. In the present study, the flow medium is also argon gas in the standard condition, and the four sides of the cavity are all diffusively reflecting and have the same temperature as the gas. Three cases with different Reynolds numbers $\left(\operatorname{Re}=U_{l i d} L / v\right)$ are investigated using the D-IP method, and the computational parameters are given in Table 2. 


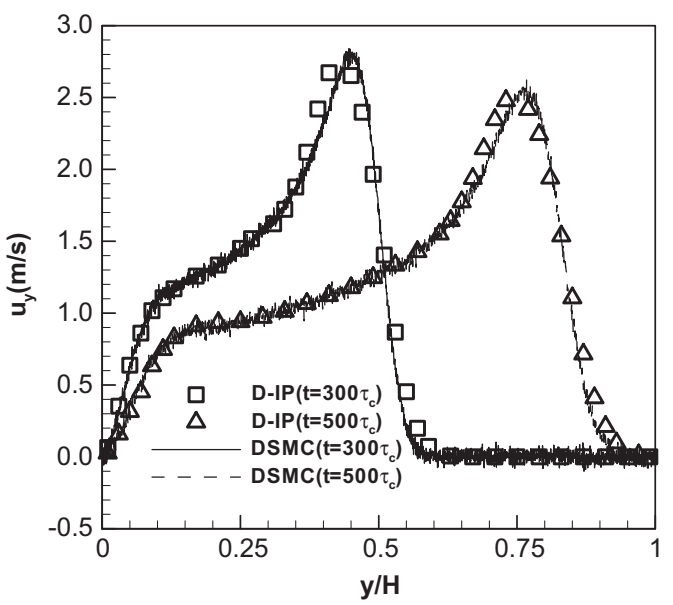

(a) Velocity

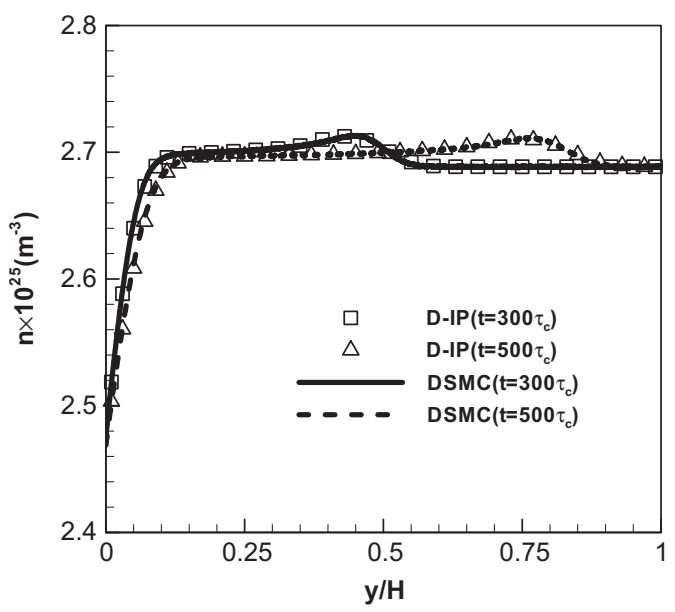

(c) Number density

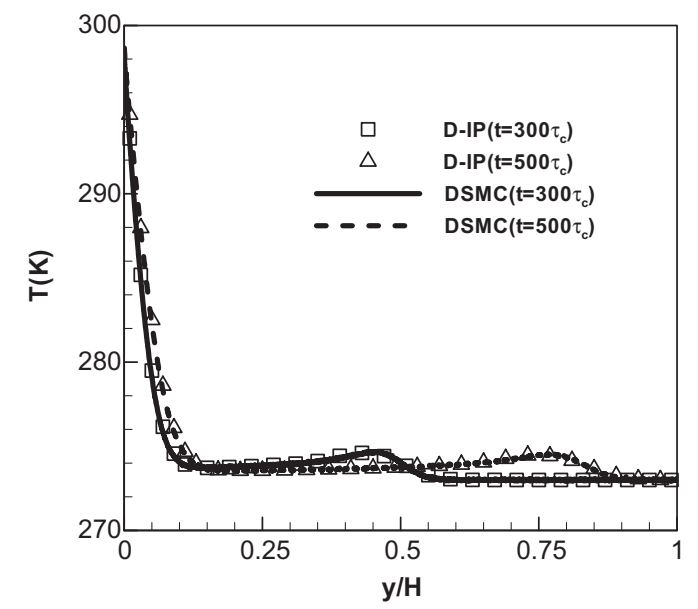

(b) Temperature

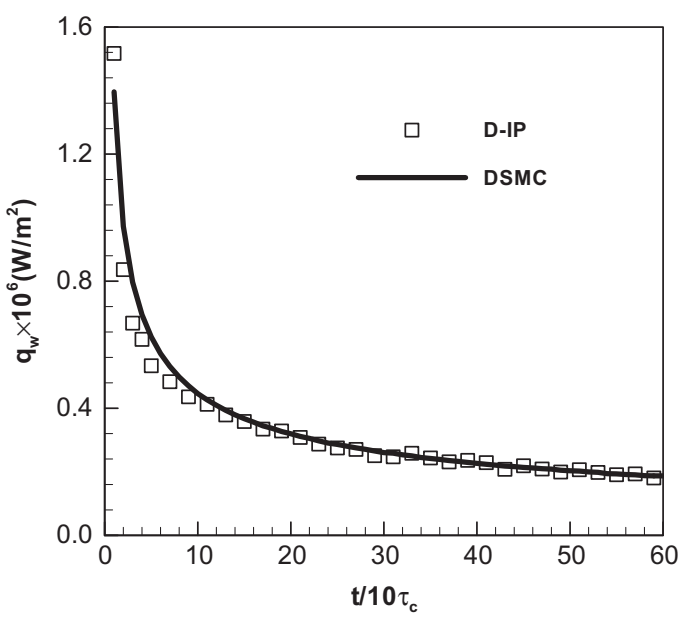

(d) Heat flux

Fig. 6. Comparison of the velocity, temperature and number density profiles at time of $300 \tau_{c}$ and $500 \tau_{c}$, as well as the evolution of heat flux, predicted by DIP and DSMC for the thermal Rayleigh flow.

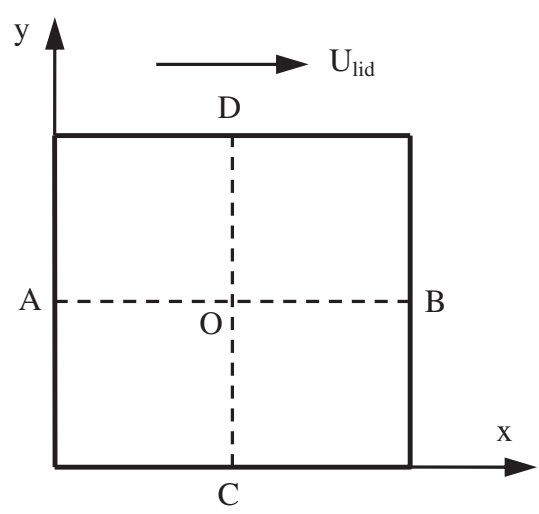

Fig. 7. Schematic diagram of a square cavity flow.

As shown in Fig. 8, as Re increase, the center positions of the main vortex move toward the geometric center of the square, and the secondary vortices become large. To verify the accuracy of the D-IP results, a quantitative comparison with the NavierStokes numerical solution of Ghia [22] has been carried out, and excellent agreement are seen clearly in Fig. 9 and Table 3. 
Table 2

Parameters used in IP computations of square cavity flows.

\begin{tabular}{llll}
\hline $\operatorname{Re}$ & 1000 & 3200 & 10000 \\
\hline$L$ & $5000 \lambda$ & $16000 \lambda$ & $50000 \lambda$ \\
$U_{\text {lid }}(\mathrm{m} / \mathrm{s})$ & 37.35 & 37.35 & 37.35 \\
Cell size & $20 \lambda$ & $67 \lambda$ & $100 \lambda$ \\
Time step & $20 \tau_{c}$ & $60 \tau_{c}$ & $100 \tau_{c}$ \\
\hline
\end{tabular}

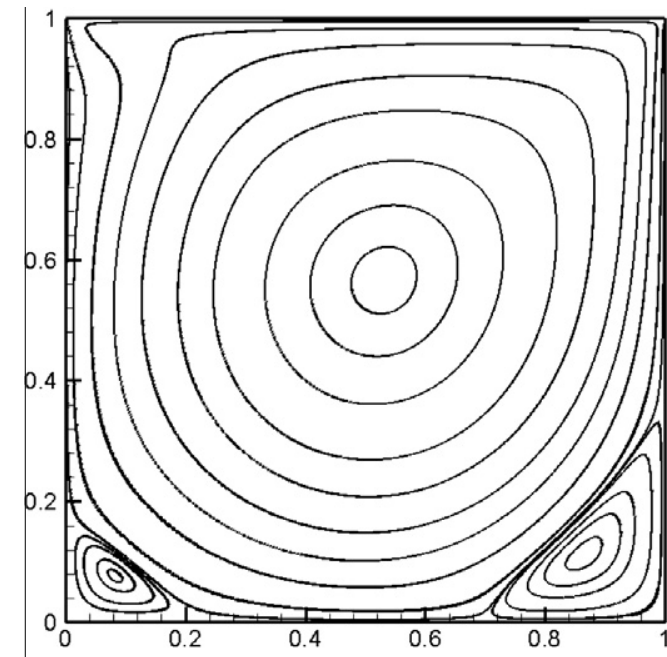

(a) $\operatorname{Re}=1000$

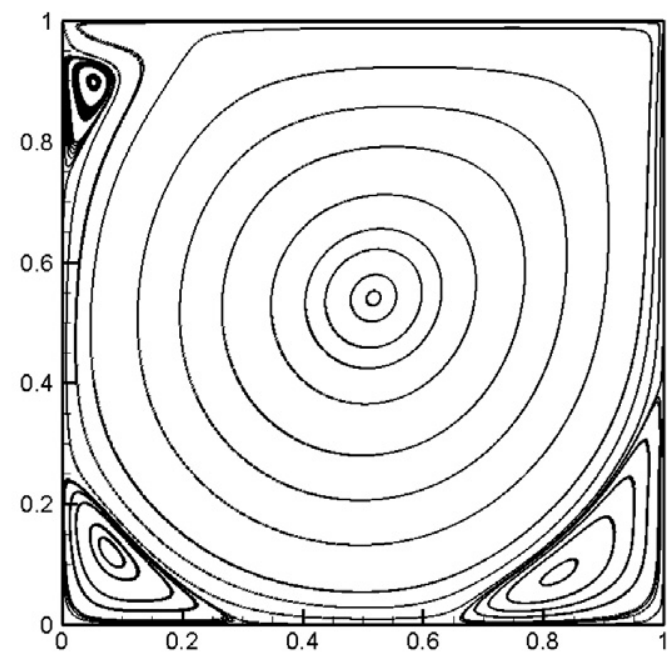

(b) $\operatorname{Re}=3200$

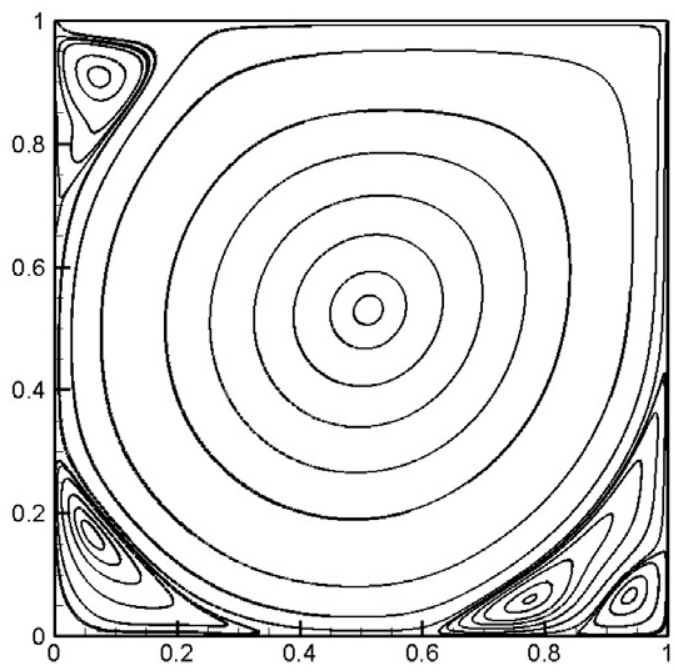

(c) $\operatorname{Re}=10000$

Fig. 8. Streamlines of the square cavity flows at three Reynolds numbers given by the D-IP method.

Compared to the N-S model, the D-IP model provides additional information on microscopic transport mechanism for various flow patterns, with the computational efficiency as a price. Does the additional information have a fine potential in solving difficult issues, e.g., laminar-turbulent transition or fully turbulent flows? This is critically important and should be a focus in further studies of the D-IP model. 

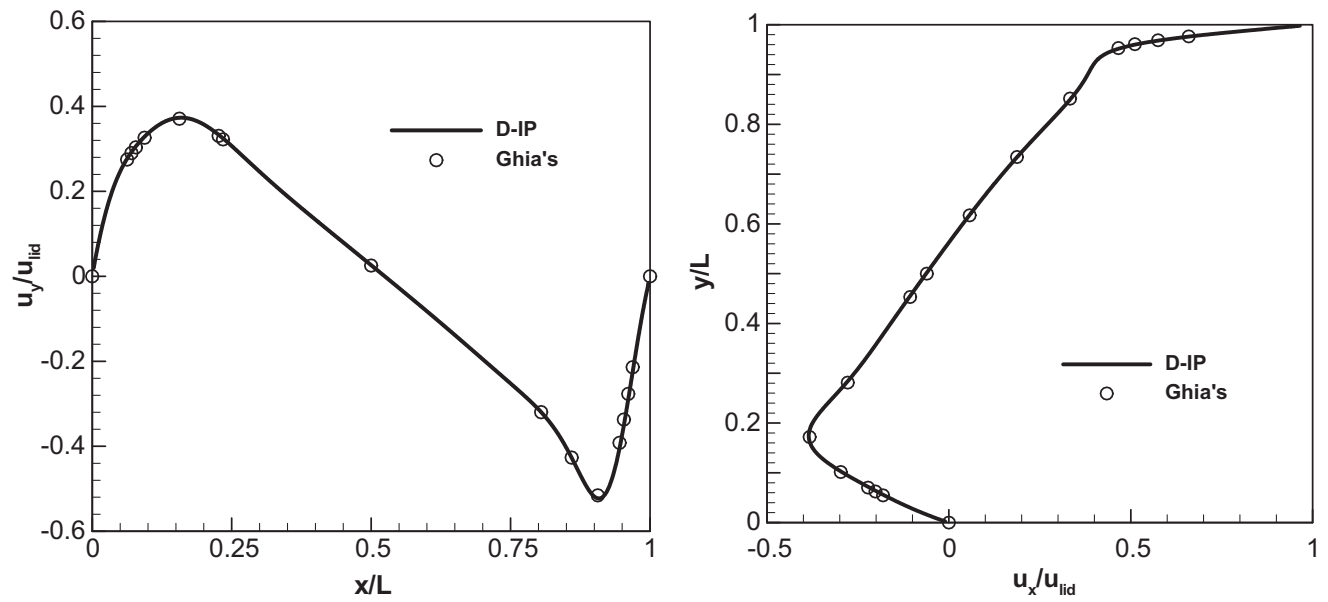

(a) $\operatorname{Re}=1000$
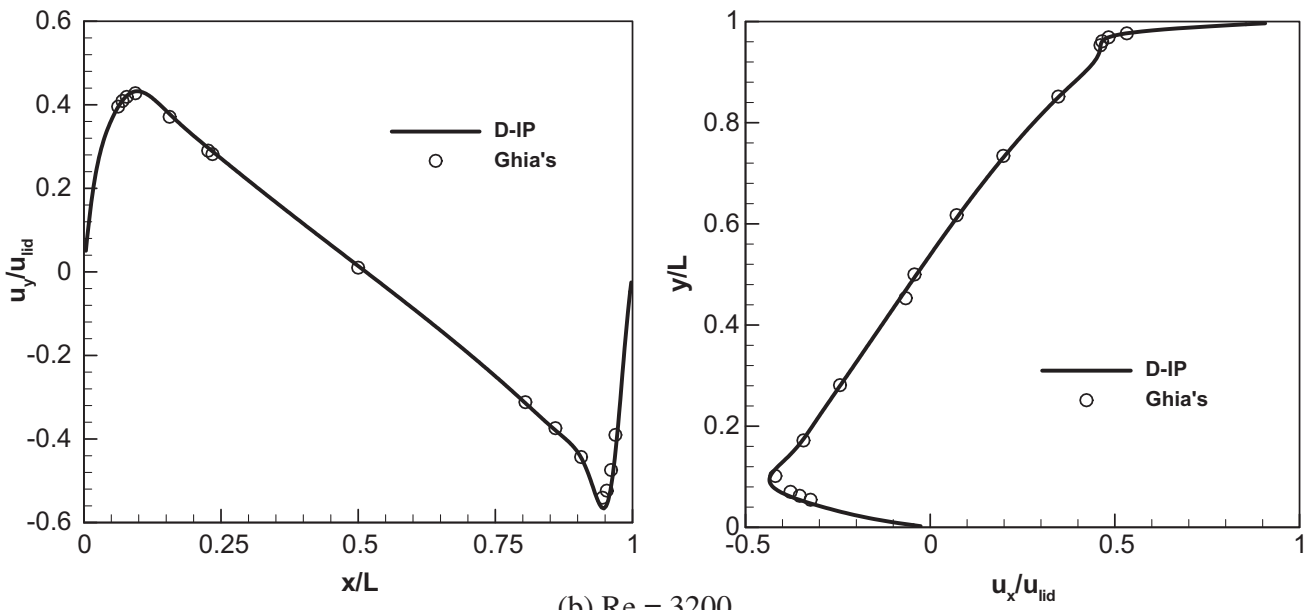

(b) $\operatorname{Re}=3200$
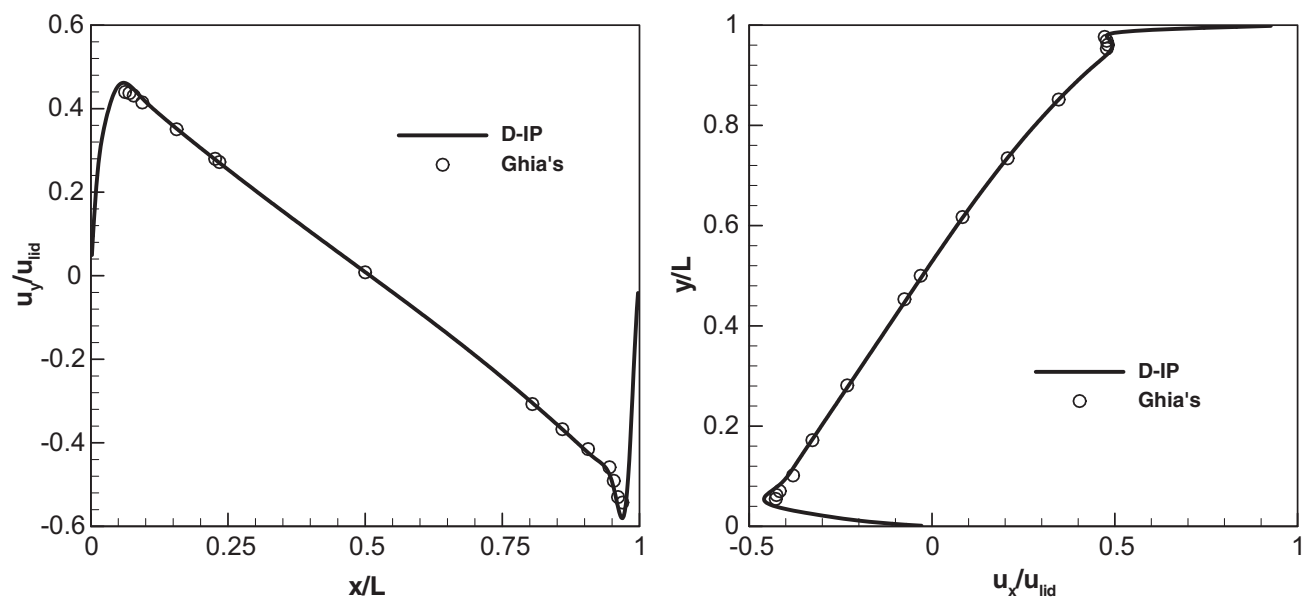

(c) $\operatorname{Re}=10000$

Fig. 9. Perpendicular velocity profiles along $A O B$ (left) and Horizontal velocity profiles along COD (right) in the square cavity flows at three Reynolds numbers. Solid line: the present results obtained by the D-IP method; circle: the Navier-Stokes numerical solutions by Ghia [22]. 
Table 3

Comparison of main vortex centers in square cavity flows at different Reynolds numbers.

\begin{tabular}{llll}
\hline Re & 1000 & 3200 & 10000 \\
\hline$x / L$ (IP) & 0.53 & 0.52 & 0.51 \\
$x / L$ (Ghia) & 0.54 & 0.52 & 0.51 \\
$y / L$ (IP) & 0.57 & 0.54 & 0.53 \\
$y /$ (Ghia) & 0.56 & 0.55 & 0.53 \\
\hline
\end{tabular}

Table 4

Compuattaional parameters of D-IP for two-dimensional channel flows.

\begin{tabular}{llllll}
\hline Case & $H / \lambda$ & $L / \lambda$ & $P_{\text {in }}(\mathrm{atm})$ & Cell number & Time step \\
\hline I & 200 & $2 \times 10^{4}$ & 1.3 & $200 \times 20$ & $10 \tau_{c}$ \\
II & 10000 & $1 \times 10^{6}$ & 1.006 & $56 \times 50$ & $200 \tau_{c}$ \\
\hline
\end{tabular}
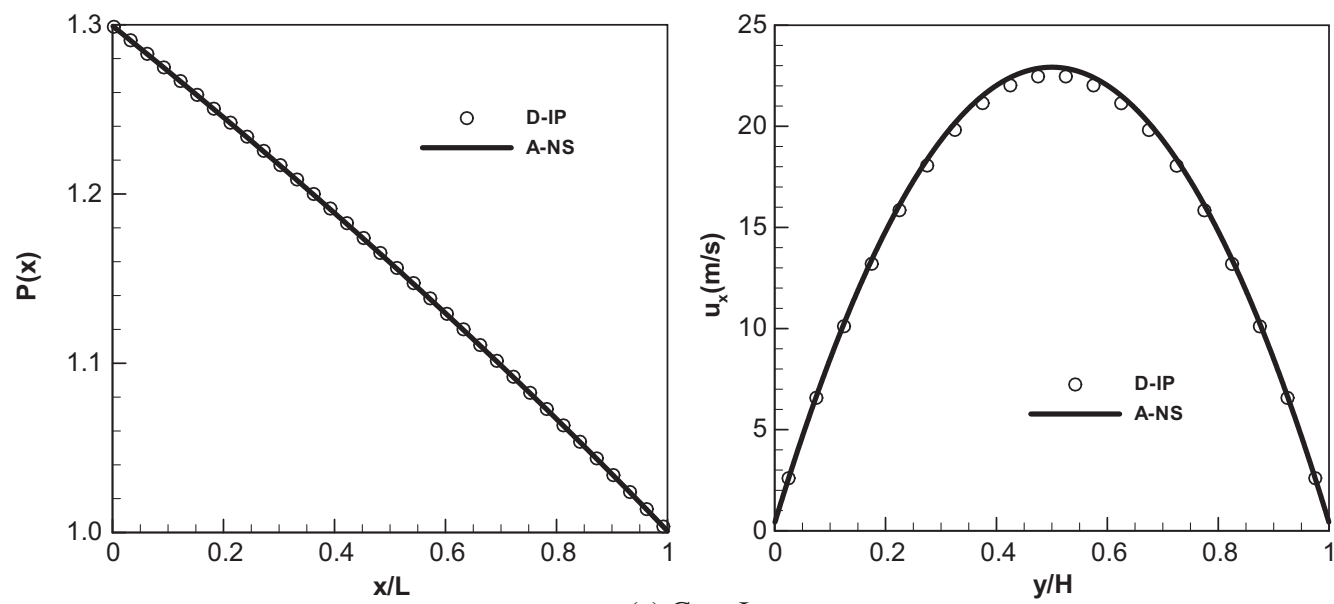

(a) Case I
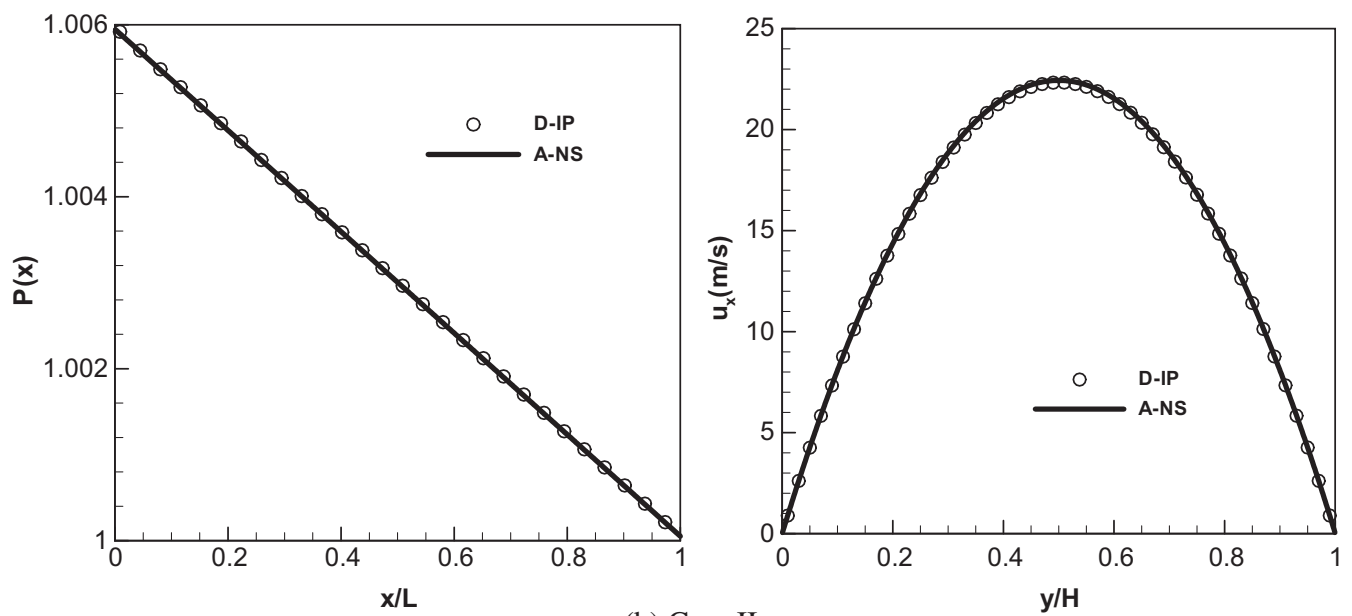

(b) Case II

Fig. 10. Pressure distributions along the streamwise direction (left) and streamwise velocity profiles at $x / L=0.5$ (right) for the channel flows. Circles: D-IP results; lines: analytical Navier-Stokes solutions [23].

\subsection{Two-dimensional channel flow}

A two-dimensional channel flow is a steady flow confined between two finite parallel plates. It is driven by a pressure difference between the inlet and outlet. In the present study, the flow medium is also argon gas, and the ratio of the channel 


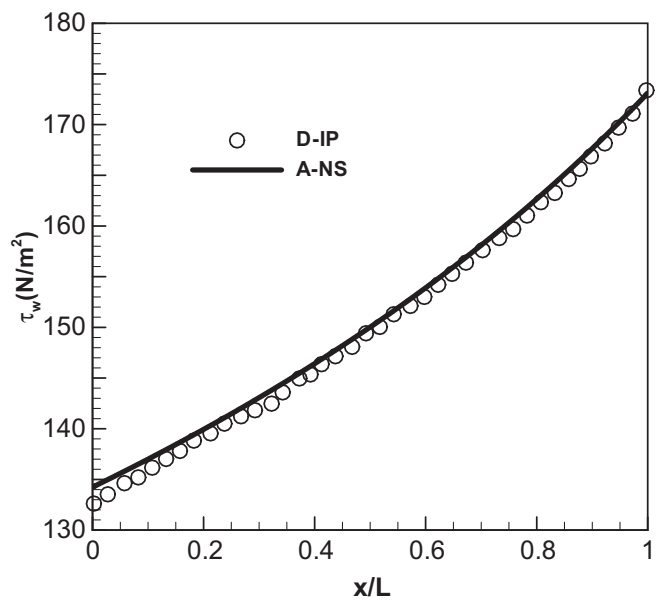

(a) Case I

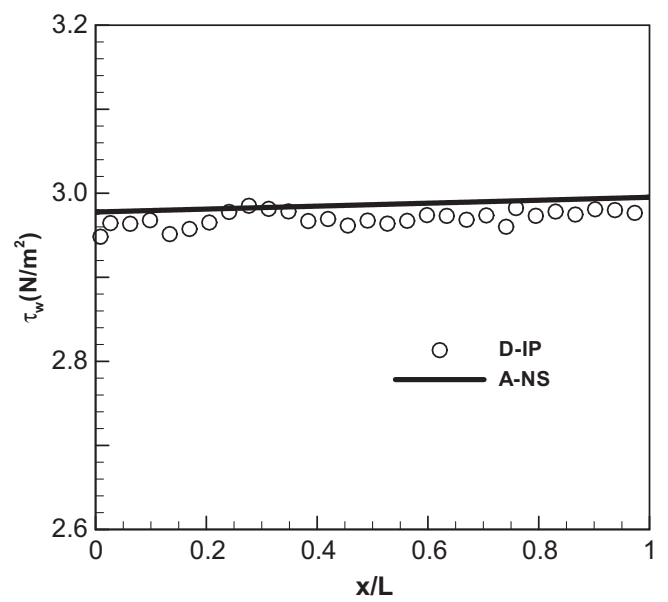

(b) Case II

Fig. 11. Shear stress distributions along the channel walls. Circles: D-IP results; lines: analytical N-S solutions [23].

length $(L)$ to its height $(H)$ is 100 . The channel walls are difussively reflecting that have the same temperature of $273 \mathrm{~K}$. Two cases are investigated, and the corresponding computational paramters are given in Table 4 . The outlet pressures for both cases are of $1 \mathrm{~atm}$, while the inlet temperatures keep at $273 \mathrm{~K}$.

Fig. 10 shows the pressure distributions in the streamwise direction and the streamwise velocity profiles obtained by D-IP for the two cases. They agree well with the analytical Navier-Stokes solutions of Arkilic et al. [23], so do the shear stress distributions along the channel walls (Fig. 11). Note that the channel length of Case II is about $6 \mathrm{~cm}$. This exhibits the DIP method capable of analyzing gas flows with a common size.

\section{Conclusions}

The D-IP method has been developed that releases the limitations of time step and cell size associated with conventional IP calculations of small Knudsen number flows, and enables us to investigate effectively the kind of flows at molecular level. For benchmark problems such as isothermal unidirectional flows, thermal Couette flow, thermal Rayleigh flow, square cavity flows, and two-dimensional channel flows with $\mathrm{Kn} \sim 10^{-3}-10^{-4}$, the D-IP calculations are not only efficient, but agree well with the DSMC or Navier-Stokes solutions. It is looking forward to applying the D-IP method to more issues like flow instability and turbulence to promote our understanding of the microscopic nonequilibrium mechanism behind these macroscopic nonlinear phenomena.

\section{Acknowledgements}

This work was supported by the National Natural Science Foundation of China under Grant No. 10921062. 


\section{Appendix A. Criterion on a simulated particle interacting with a computational boundary}

According to the diffusion model, when simulator $k$ has no interaction with a computational boundary during a time step, its new position and thermal velocity after $\Delta t$ are determined by Eqs. (4) and (6), respectively. Now let us consider an interaction between a simulator and a computational boundary. This consists of two issues. One is to judge whether a simulator interacts with a computational boundary; the other is how to determine the molecular position and velocity after an interaction. The second issue is the same as the conventional IP method, we will focus on the first one.

For the sake of simplicity, a one-dimensional case is considered. For a computational boundary at $y=0$, there are two situations:

(1) Deterministic interaction. If the position of simulator $k$ after a time step is smaller than zero, it interacts with the boundary during the interval $\Delta t$.

(2) Probabilistic interaction. If the position of simulator $k$ after a time step is larger than zero, it still probably interacts with the boundary during $\Delta t$. Our problem can be stated as follows: for a known position $y_{0}>0$ at $t$, what is the probability $f_{\text {out }}\left(y, y_{0}\right)$ in which $y$ is always larger than zero during $\Delta t$ ?

Such a problem was studied well, e.g. in [21], which gave rise to

$$
f_{\text {out }}\left(y, y_{0}\right)=\frac{1}{(2 \pi F)^{\frac{1}{2}}}\left\{\exp \left[\frac{\left(y-\left(y_{0}+d_{y 0}\right)\right)^{2}}{2 F}\right]-\exp \left[\frac{\left(y+2 \alpha \lambda_{B}+\left(y_{0}+d_{y 0}\right)\right)^{2}}{2 F}\right]\right\},
$$

where $d_{y 0}=c_{y}^{\prime}(t)\left(1-e^{-\zeta t}\right) / \zeta, \alpha=1.46, \lambda_{B}=\sqrt{k_{B} T /\left(m \zeta^{2}\right)}$, and $F$ is defined by Eq. (3c).

According to the Chandrasekhar distribution (2), the probability from $y_{0}$ to $y$ is

$$
f\left(y, y_{0}\right)=\frac{1}{(2 \pi F)^{1 / 2}} \exp \left\{\frac{\left[y-\left(y_{0}+d_{y 0}\right)\right]^{2}}{2 F}\right\} .
$$

Compare the ratio of $f_{\text {out }}\left(y, y_{0}\right)$ to $f\left(y, y_{0}\right)$ to a random number uniformly distributed between 0 and 1 . If the ratio is less than the random number, the simulator interacts with the boundary during the time interval; otherwise, no interaction.

\section{References}

[1] G.A. Bird, Molecular Gas Dynamics and Direct Simulation of Gas Flows, Clarendon Press, Oxford, 1994.

[2] E.S. Oran, C.K. Oh, Z.C. Cybyk, Direct simulation Monte Carlo: recent advances and applications, Ann. Rev. Fluid Mech. 30 (1998) $403-441$.

[3] G. Pham-Van-Diep, D. Erwin, E.P. Muntz, Nonequilibrium molecular motion in a hypersonic shock wave, Science 245 (1989) $624-626$.

[4] M.S. Ivanov, S.F. Gimelshein, Computational hypersonic rarefied flows, Ann. Rev. Fluid Mech. 30 (1998) 469-505.

[5] F.J. Alexander, A.L. Garcia, B.J. Alder, Cell size dependence of transport coefficients in stochastic particle algorithms, Phys. Fluids 10 (1998) $1540-1542$.

[6] N.G. Hadjiconstantinou, Analysis of discretization in the direct simulation Monte Carlo method, Phys. Fluids 12 (2000) $2634-2638$.

[7] P. Jenny, M. Torrilhon, S. Heinz, A solution algorithm for the fluid dynamic equations based on a stochastic model for molecular motion, J. Comput. Phys. 229 (2010) 1077-1098.

[8] M.H. Gorji, M. Torrilhon, P. Jenny, Fokker-Planck model for computational studies of monatomic rarefied gas flows, J. Fluid Mech. 680 (2011) 574-601.

[9] J. Fan, C. Shen, Statistical simulation of low-speed unidirectional flows in transition regime, in: R. Brun (Ed.), Rarefied Gas Dynamics, Cepadus-Editions, Toulouse, 1999, pp. 245-252.

[10] J. Fan, C. Shen, Statistical simulation of low-speed rarefied gas flows, J. Comput. Phys. 167 (2001) 393-412.

[11] C.P. Cai, I.D. Boyd, J. Fan, G.V. Candler, Direct simulation methods for low-speed microchannel flows, J. Thermophys. Heat Trans. 14 (2000) $368-378$.

[12] C. Shen, J. Fan, C. Xie, Statistical simulation of rarefied gas flows in microchannels, J. Comput. Phys. 189 (2003) 512-526.

[13] Q. Sun, I.D. Boyd, Drag on a flat plate in low-Reynolds number gas flows, AIAA J. 42 (2004) 1066-1072.

[14] Q. Sun, I.D. Boyd, Flat plate aerodynamics at very low Reynolds numbers, J. Fluid Mech. 502 (2004) 199-206.

[15] Q. Sun, I.D. Boyd, Theoretical development of the information preservation method for strongly nonequilibrium gas flows, AIAA Pap. (2005) 20054828.

[16] N.D. Masters, W. Ye, Octant flux splitting information preservation DSMC method for thermally driven flows, J. Comput. Phys. 226 (2007) $2044-2062$.

[17] J. Zhang, J. Fan, J. Jiang, Multiple temperature model for the information preservation method and its application to nonequilibrium gas flows, J. Comput. Phys. 230 (2011) 7250-7265.

[18] S. Chandrasekhar, Stochastic problems in physics and astronomy, Rev. Mod. Phys. 15 (1943) 1-89.

[19] R. Zwanzig, Nonequilibrium Statistical Mechanics, Oxford University Press, New York, 2001.

[20] S. Heinz, Molecular to fluid dynamics: the consequences of stochastic molecular motion, Phys. Rev. E 70 (2004) 036308.

[21] P.S. Hagan, C.R. Doering, C.D. Levermore, The distribution of exit times for weakly colored noise, J. Stat. Phys. 54 (1989) $1321-1352$.

[22] U. Ghia, K.N. Ghia, C.T. Shin, High-Re solutions for incompressible flow using the Navier-Stokes equations and a multigrid method, J. Comput. Phys. 48 (1982) 387-411.

[23] E.B. Arkilic, M.A. Schmidt, K.S. Breuer, Gaseous slip flow in long microchannels, J. MEMS 6 (1997) 167-178. 